\title{
LHC PHYSICS
}

\author{
Felicitas Pauss \\ Institute for Particle Physics (IPP), ETH Zürich, \\ CH-8093 Zürich, Switzerland \\ felicitas.pauss@cern.ch
}

AbstraCt: The Large Hadron Collider (LHC) at CERN will provide proton-proton collisions at a centre-of-mass energy of $14 \mathrm{TeV}$ with a design luminosity of $10^{34} \mathrm{~cm}^{-2} \mathrm{~s}^{-1}$. The exploitation of the rich physics potential offered by the LHC will be illustrated using the expected performance of the two general-purpose detectors ATLAS and CMS.

The detector design requirements necessary to extract the physics under the challenging experimental conditions at the LHC are discussed. This is followed by an analysis of search methods for the Higgs sector and the detection of supersymmetric particles.

\section{Introduction}

The Standard Model (SM) of particle physics the theory of electroweak and strong forces - provides a remarkably successful theoretical picture [i]. The SM has been tested rigorously at LEP, the Tevatron and the linear collider at SLAC. The four LEP experiments have already given a definitive answer to the number of fundamental building blocks of matter: there exist three families of quarks and leptons with a light neutrino.

One of the key questions in particle physics today is the origin of the spontaneous symmetry breaking mechanism. The electroweak sector of the SM postulates that the Higgs mechanism is responsible for this symmetry breaking, and predicts a scalar Higgs boson. Introducing this Higgs boson in the SM allows the masses of all particles to be expressed in terms of their couplings to the Higgs. In order to complete the SM prediction we therefore have to establish experimentally the existence of the last missing element: the Higgs boson [i27].

Even though the SM describes existing data very well, and even if it successfully passes further tests, we know that this Model is incomplete, as it supplies no answer to some fundamental questions. One problem of the SM is the instability of the mass of an elementary scalar, such as the Higgs boson, under radiative corrections in the presence of a high scale, like for example the Planck scale $\left(\approx 10^{19} \mathrm{GeV}\right)$. These divergences disappear in Supersymmetry (SUSY) because of cancellations between the virtual effects of SM particles and their supersymmetric partners, which are introduced to every known fermion and boson of the SM. Furthermore, SUSY must be a broken symmetry because known particles have no super-partner of the same mass. These must be heavier, and are therefore not yet discovered.

Another problem originates from extrapolating the coupling strength of fundamental forces measured at mass scales of a few $100 \mathrm{GeV}$ to energy scales relevant for cosmology, i.e. energies of about $10^{15}$ to $10^{19} \mathrm{GeV}$. Performing this extrapolation within the SM does not lead to unification of forces at very high scales. Introducing however SUSY unification of the electromagnetic, weak and strong forces at the GUT scale $\left(\approx 10^{15} \mathrm{GeV}\right)$ is predicted which is consistent with a SUSY mass scale of $\mathcal{O}(\mathrm{TeV})$.

It is possible that the Higgs boson is an elementary particle as predicted in the SM and its supersymmetric extension. Alternatives to a fundamental scalar Higgs involve new strong forces. In models without a scalar Higgs, the $\mathrm{W}$ and $\mathrm{Z}$ masses could then be due to a dynamical 


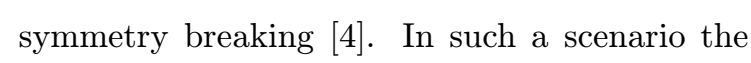
symmetry breaking could lead to a strong interaction between the longitudinal components of the intermediate vector bosons $\left(\mathrm{W}_{L}, \mathrm{Z}_{L}\right)$. This strong interaction may be resonant or not. Resonances may occur in analogy with $\pi \pi$-scattering, which leads to $\operatorname{spin}=1 \rho$-like states, or spin $=0$ very broad resonances, expected to be in the $\mathrm{TeV}$ mass range.

In spite of the impressive success of the SM there is a general consensus that the SM is not the ultimate description of nature, and that new phenomena should manifest themselves in the energy region of order $1 \mathrm{TeV}$. The Large Hadron Collider (LHC), operating at a centre-of-mass energy of $14 \mathrm{TeV}$ with a design luminosity of $10^{34} \mathrm{~cm}^{-2} \mathrm{~s}^{-1}$, will be the first machine to probe parton-parton collisions directly at energies $\approx 1$ $\mathrm{TeV}$ [5.5. Such energies will be essential to address, for example, the questions of the origin of spontaneous symmetry breaking.

Currently no experimental evidence exists for any new phenomena. We will focus in these lectures mainly on searches for the Higgs and for Supersymmetry. The ideas and methods presented should nevertheless provide a good guidance for searches of more 'exotic' physics channels.

\section{Present Experimental Status of the Standard Model and Beyond}

In the following we summarise briefly the present status of physics topics relevant for LHC and speculate about what one might know from future experimental results before the start-up of the LHC, foreseen in the year 2005.

It is well known that the value of the Higgs mass is not predictable within the SM. On the other hand, the Higgs cannot be too heavy, otherwise the perturbative regime breaks down, and this leads to an upper bound on the Higgs mass of about $1000 \mathrm{GeV}$.

The requirement of perturbative consistency of the theory up to a scale $\Lambda$ sets an upper bound on the SM Higgs mass, while arguments of vacuum stability suggest a lower Higgs mass limit [i-i]', depending also strongly on the top mass. Taking the measured value of the top mass $\left(\mathrm{m}_{t}=\right.$ $174.1 \pm 5 \mathrm{GeV}$ ) and assuming that no new physics

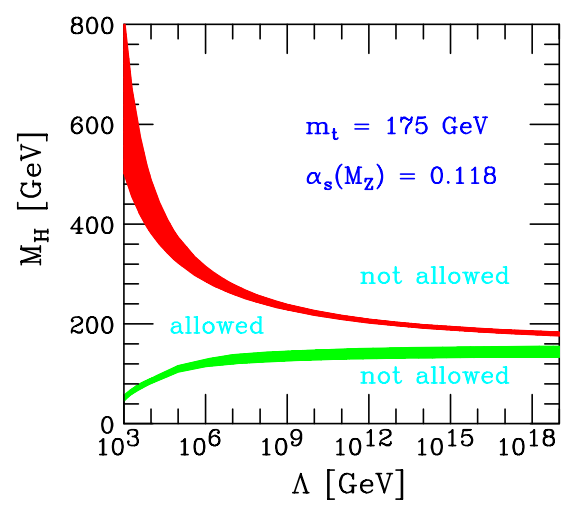

Figure 1: The area between the two curves shows the allowed Higgs mass range assuming the validity of the Standard Model up to a scale $\Lambda$ [6;i].

exists below the Planck scale, the Higgs mass should be around $160 \pm 20 \mathrm{GeV}$, as shown in figure 1.

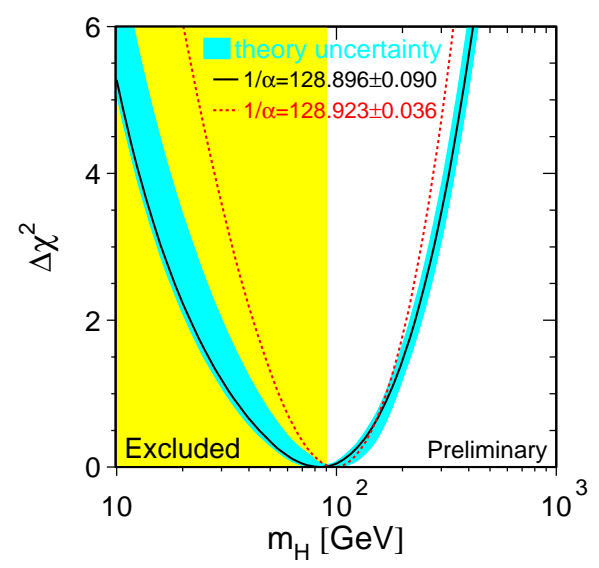

Figure 2: $\Delta \chi^{2}$ result of a fit to all electroweak observables assuming to have the Higgs mass as the only remaining free parameter ["].".

Particle physicists have been searching for many years for the Higgs boson, from zero mass up to the highest masses accessible at existing particle accelerators. At present, the four LEP experiments have ruled out the existence of a Higgs with a mass of less than $95 \mathrm{GeV}[i \overline{]}$. From global fits to electroweak data one obtains an upper limit on the Higgs mass of $280 \mathrm{GeV}(95 \%$ C.L.), as shown in figure 2 [is]

By the end of the year 2000 one expects to discover at LEP200 a Higgs boson up to $m_{H} \approx$ $106 \mathrm{GeV}$, assuming $150 \mathrm{pb}^{-1}$ per experiment at $\sqrt{s}=200 \mathrm{GeV}$. In case no Higgs signal is found, a mass limit of $\approx 109 \mathrm{GeV}$ (95\% C.L.) will be 
placed [ing.

In the supersymmetric extension of the SM a set of new particles should exist with a mass scale around $1 \mathrm{TeV}$. The minimal version of the supersymmetric SM (MSSM) contains three neutral and two charged Higgs bosons. One of the neutral ones is expected to have a mass around $100 \mathrm{GeV}$ and is therefore of particular interest for searches at LEP200. So far the searches for this lightest Higgs boson resulted in a lower mass limit of about $80 \mathrm{GeV}$. The expectation for SUSY Higgs searches at LEP200 is summarised in figure $3[1 \overline{1} 0$.$] . However, the discovery potential at$ LEP200 depend strongly on the available energy. Thus few GeV increase in $\sqrt{s}$ could change our understanding of the Higgs sector dramatically.
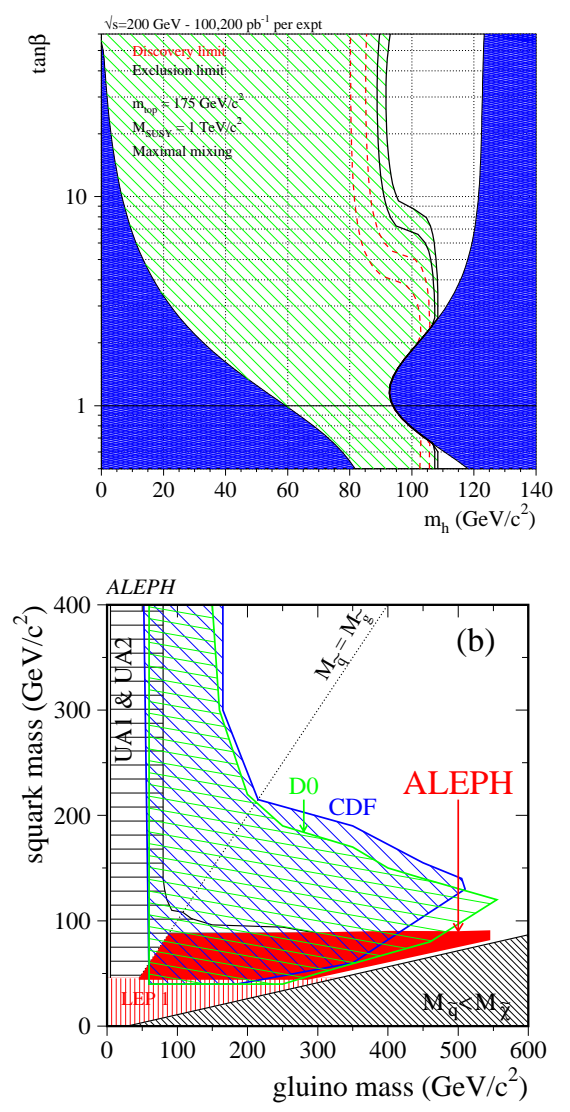

Figure 3: Expected sensitivity of SUSY Higgs searches (top) at LEP200 $(\sqrt{s}=200 \mathrm{GeV})$ and recent lower mass limits for squarks and gluinos (bottom) [10].

Direct searches for sparticles at LEP200 have reached in most cases the kinematical limit, i.e. sparticle masses below $\approx 90 \mathrm{GeV}$ are excluded [i] 001$]$. Searches for sparticles at the Tevatron have excluded gluino and squark masses below about 250 $\mathrm{GeV}$, as shown in figure 3. With the data collected during RunII $\left(\approx 1 \mathrm{fb}^{-1}\right.$ /year $)$ at the Tevatron, scheduled to start in 2000 , one expects to reach gluino and squark masses of 300 to 400 $\mathrm{GeV}$.

\section{The World of Physics at LHC}

Discovering new phenomena in high-energy physics experiments rely on the capability to separate new from known phenomena. The methods used exploit the different kinematics of signals and backgrounds in searching for new mass peaks, or comparing $\mathrm{p}_{T}$ spectra of leptons, photons and jets and their angular correlations with SM predictions. Other searches exploit the missing transverse energy signature which might originate from neutrinos or neutrino-like objects, or simply from detector imperfections. Depending on the particular physics process, different aspects of the detector performance parameters are important. The search for mass peaks requires in general excellent energy and momentum resolution for individual particles. Searches based on the missing transverse energy signature require detectors with hermetic calorimeter coverage up to $|\eta|=5$.

Although the most exciting discoveries will be those of totally unexpected new particles or phenomena, one can only demonstrate the discovery potential of the proposed experiments using predicted new particles. However, the experiments designed under these considerations should also allow the discovery of whatever new phenomena might occur in multi- $\mathrm{TeV}$ pp collisions.

\subsection{The Experimental Challenge at LHC}

The total cross-section at hadron colliders is very large, i.e. about $100 \mathrm{mb}$ at the $\mathrm{LHC}$, resulting in an interaction rate of $\approx 10^{9} \mathrm{~Hz}$ at the design luminosity. Figure 4 shows the expected energy dependence of the total cross-section and of some interesting physics processes which have much smaller cross sections. The detection of processes with signal to total cross-section ratios of about $10^{-12}$, as for example for a $100 \mathrm{GeV}$ 
Higgs decaying into two photons, will be a difficult experimental challenge.

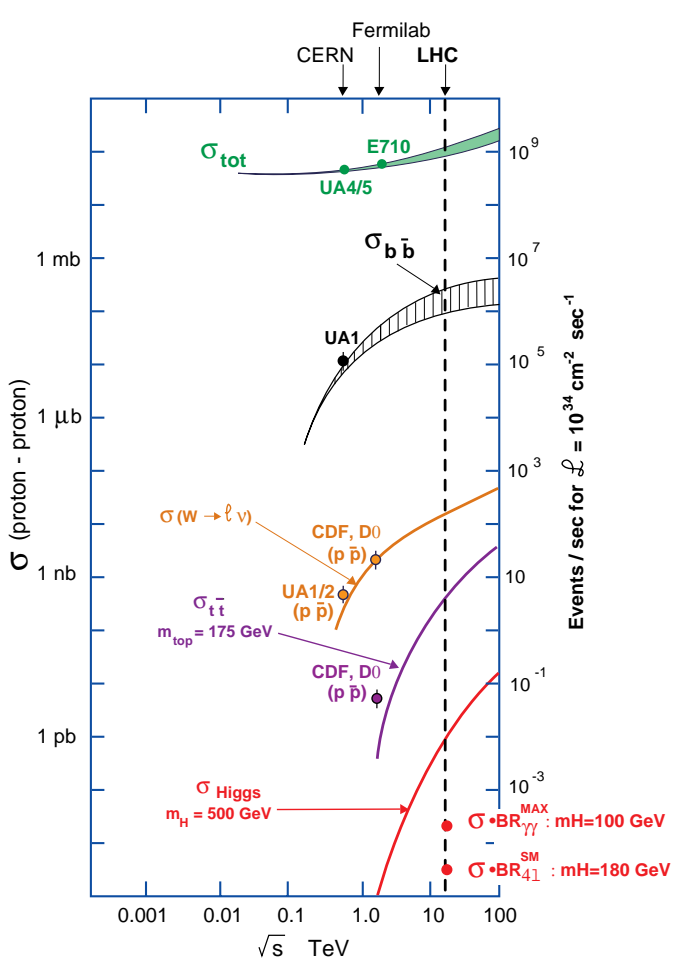

Figure 4: Energy dependence of some characteristic cross-sections at hadron colliders.

Many of the above mentioned new particles decay into $\mathrm{W}$ and $\mathrm{Z}$ bosons, charged leptons or photons. Ws and Zs will have to be detected through their leptonic decays because hadronic decay modes will be overwhelmed by the QCD background. These purely leptonic modes lead to very small branching fractions. In order to observe such signals, a machine with high constituent centre-of-mass energy and high luminosity is required.

The LHC fulfils these requirements, but the high luminosity leads to difficult experimental conditions: with an inter-bunch crossing time of $25 \mathrm{~ns}$ at design luminosity, on average 20 interactions ("minimum bias events") are expected per crossing, resulting in about 1000 charged tracks every $25 \mathrm{~ns}$, in the pseudorapidity range of $|\eta| \leq$ 3 . Therefore, at peak luminosity, on average 2.2 charged particles are expected every $25 \mathrm{~ns}$ in a $2 \times 2 \mathrm{~cm}^{2}$ cell at a distance of $7.5 \mathrm{~cm}$ from the interaction point at $\eta=0$. This example shows that the inner tracking detectors have to oper- ate in a hostile environment. Such high particle fluxes will make track reconstruction difficult. Simulation results make us believe that a very large number of electronic channels and good time resolution should nevertheless guarantee a high track-finding efficiency.

The expected $10^{9}$ inelastic pp events per second at design luminosity will also generate a hostile radiation environment. This results in high radiation levels (high integrated dose) and in a large flux of low energy neutrons in the experimental area. Radiation hard detectors and electronics are therefore required. Induced activity in the forward calorimeters has to be taken into account for long-term access and maintenance.

\section{Design Objectives of ATLAS and CMS}

An important aspect of the overall detector design is the magnetic field configuration. Large bending power is required to measure precisely high-momentum muons and other charged particles. The choice of the magnet structure strongly influences the remaining detector design.

A solenoid provides bending in the transverse plane and thus facilitates the task of triggering on muons, which are pointing to the event vertex, so that one can take advantage of the small transverse dimensions of the beam $(20 \mu \mathrm{m})$. A drawback of a solenoid with limited length is the degradation of momentum resolution in the forward direction; therefore either a very long solenoid is required or an endcap toroid system has to be added. The main advantage of a toroid is a constant $\mathrm{p}_{T}$ resolution over a wide rapidity range. However, the closed configuration of a toroid does not provide magnetic field for the inner tracking, thus an additional solenoid is required to measure the momenta of charged tracks in the inner tracking detectors.

The identification and precise measurement of electrons, photons and muons over a large energy range, complemented by measurements of jets and missing transverse energy are the basic design goals of the ATLAS and CMS detectors. In addition, a good impact-parameter resolution and secondary vertex reconstruction will be important for b-tagging. 
The ATLAS [1] 1 1. magnet configuration based on a superconducting air-core toroid, complemented by a superconducting solenoid of $2 \mathrm{~T}$ around the inner tracking detectors. The thin solenoid is followed by a high-granularity liquid-argon sampling calorimeter. In the toroidal magnet configuration the muon triggering, identification and precision measurement can be entirely performed in the muon spectrometer, without using the inner detectors.

The CMS 1' 1 in detector will use a high-field superconducting solenoid $(4 \mathrm{~T})$ allowing for a compact design of the muon spectrometer. The inner coil radius of about $3 \mathrm{~m}$ is large enough to accommodate the inner tracking system and the calorimeters. For the electromagnetic calorimeter $\mathrm{PbWO}_{4}$ crystals have been chosen.

The hadron calorimeter (also located before the coil) consists of copper absorber plates and scintillator tiles. Muons are triggered, identified and measured in four identical muon stations inserted in the return yoke. Their momenta are measured independently in the inner tracking chambers to improve the overall momentum measurement. More details about the subdetector requirements and performance figures can be found in reference $\left[i \overline{1} \overline{3}_{-}^{\prime}\right]$.

\section{TeV-scale Physics at LHC}

Exploiting the LHC physics potential means that we can answer or shed considerable light on fundamental open questions such as the mass problem or unification of fundamental interactions. In the following section we discuss first the Higgs sector, followed by selected topics in sparticle searches in order to demonstrate the discovery potential of the proposed pp detectors ${ }^{1}$.

\subsection{SM Higgs search}

Figure 5 shows the next-to-leading order Higgs cross-sections [1 14] at the LHC for various pro-

\footnotetext{
${ }^{1}$ One usually assumes that "one" LHC year with a peak luminosity of $\mathrm{L}=10^{33} \mathrm{~cm}^{-2} \mathrm{~s}^{-1}$ and a running time of $10^{7} \mathrm{~s}$ produces an integrated luminosity of $10 \mathrm{fb}^{-1}$. A more realistic estimate would use an average run luminosity and includes losses due to machine and detector efficiencies. It would thus be more conservative to assume that a running time of $10^{7} \mathrm{~s}$ per year with the initial luminosity requires about $2-3$ years to accumulate $10 \mathrm{fb}^{-1}$.
}

duction processes as a function of the Higgs mass. By far the largest contribution comes from the gluon-gluon fusion process [1] ${ }_{1}^{1}$. Depending on the Higgs mass, its detection involves several different signatures. The Higgs search is therefore an excellent reference physics process to evaluate the overall detector performance. In particular, the search for the intermediate Higgs $\left(\mathrm{m}_{Z} \leq\right.$ $\mathrm{m}_{H} \leq 2 \mathrm{~m}_{Z}$ ) is known to pose demanding requirements on the detectors. The natural width of the Higgs in this mass range is very small. The measured width of the signal will therefore be dominated entirely by the instrumental mass resolution. Figure 6 shows the $\sigma \times B R\left[1 \overline{6}_{1}^{\prime}\right]$ for

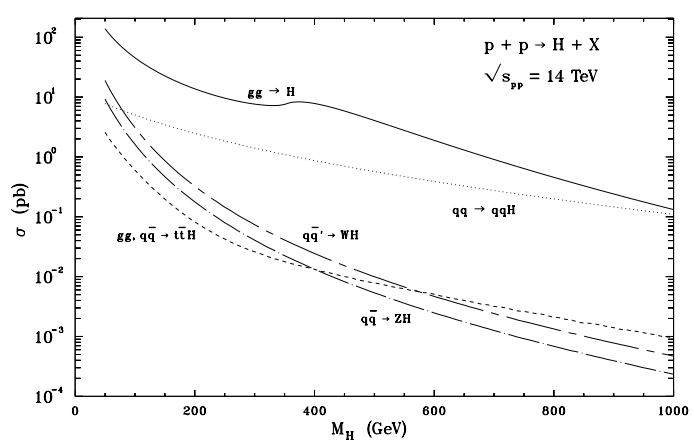

Figure 5: Next-to-leading order cross-section calculations for the SM Higgs [i] it].

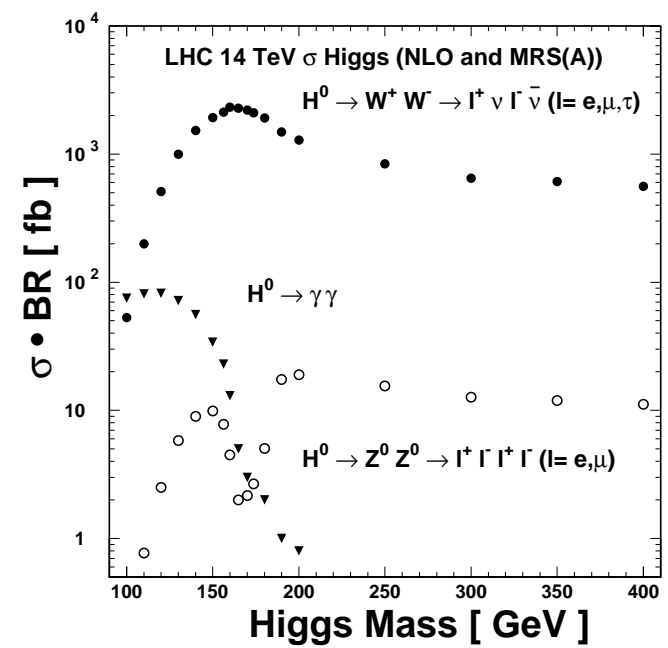

Figure 6: Expected $\sigma \times B R$ for different detectable SM Higgs decay modes [i] $\left[\begin{array}{l}- \\ 6\end{array}\right]$.

the most promising Higgs search channels: $H \rightarrow$ $\gamma \gamma, H \rightarrow Z Z^{(*)} \rightarrow 4 \ell^{ \pm}$, and $H \rightarrow W W^{(*)} \rightarrow$ $\ell^{+} \nu \ell^{-} \bar{\nu}$ 
Figure 7 summarises the expected observability of the SM Higgs in ATLAS ${ }^{2}$ and CMS, assuming $100 \mathrm{fb}^{-1}$.
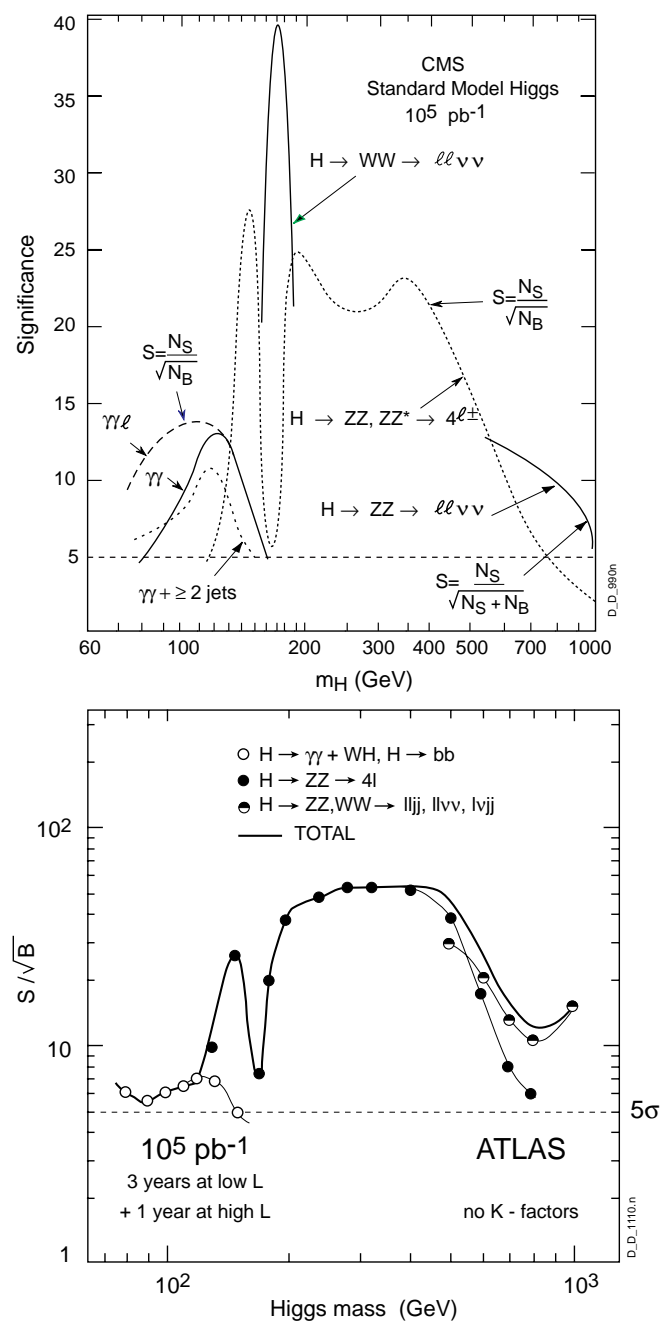

Figure 7: Expected signal significances for the SM Higgs search in ATLAS and CMS, assuming 100 $\mathrm{fb}^{-1}$.

The most promising signature for a SM Higgs with masses between the expected LEP200 limit and $130 \mathrm{GeV}$ is the decay $H \rightarrow \gamma \gamma$ with a branching ratio of only $\approx 2 \times 10^{-3}$. As can be seen from figure 8 , this signal has to be detected above a large background from continuum $\gamma \gamma$ events. The detection of such a signal requires an excellent $\gamma \gamma$ mass resolution of $\leq 1 \%$ (i.e. $\leq 1 \mathrm{GeV}$ for $\mathrm{m}_{H}=100 \mathrm{GeV}$ ) and a very good $\pi^{0}$ rejection capability. More details about this channel can

\footnotetext{
${ }^{2}$ The expected Higgs signal significance from ATLAS does not yet include the channel $H \rightarrow W W^{(*)} \rightarrow$ $\ell^{+} \nu \ell^{-} \bar{\nu}$
}

be found in section 5.1.1.
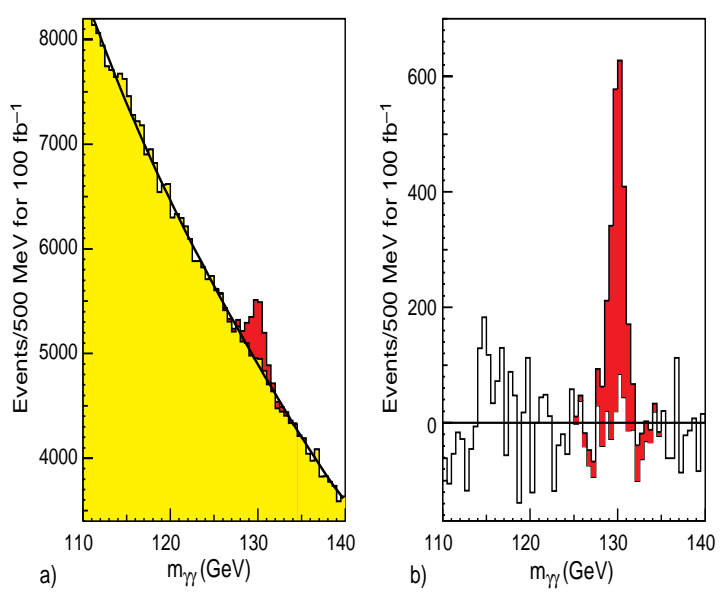

Figure 8: CMS simulation for $H \rightarrow \gamma \gamma\left(\mathrm{m}_{H}=130\right.$ $\mathrm{GeV}$ ) before and after background subtraction.

For Higgs masses between $130 \mathrm{GeV}$ and 200 $\mathrm{GeV}$ the sensitivity of the $4 \ell^{ \pm}$signature suffers from very low branching ratios as illustrated in figures 6 and much smaller signals, like the ones shown in figure 9, are expected. Consequently, a 5 standard deviation signal requires integrated luminosities of at least $30-100 \mathrm{fb}^{-1}$. A recent study has demonstrated that this Higgs mass region can also be covered by the $H \rightarrow W W\left(^{*}\right) \rightarrow$ $\ell^{+} \nu \ell^{-} \bar{\nu}$ decay [i] scribed in section 5.1.2, shows that this channel should allow to discover a SM Higgs with 5 standard deviation for a Higgs mass between 140-200 $\mathrm{GeV}$ and integrated luminosities below $5 \mathrm{fb}^{-1}$.

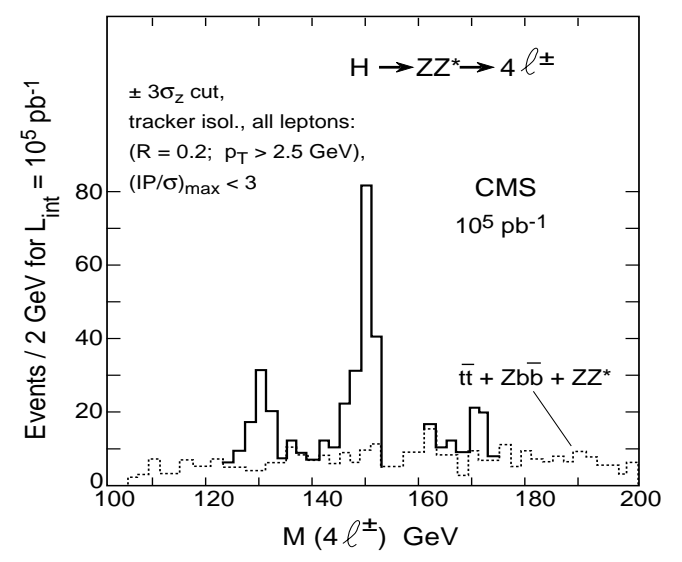

Figure 9: CMS simulation for $H \rightarrow Z Z^{*} \rightarrow$ $\ell^{+} \ell^{-} \ell^{+} \ell^{-}$and $\mathrm{m}_{H}=130,150$ and $170 \mathrm{GeV}$.

For $2 \times \mathrm{m}_{Z} \leq \mathrm{m}_{H} \leq 500 \mathrm{GeV}$ the decay $H \rightarrow$ 
$Z Z \rightarrow 4 \ell^{ \pm}$provides the experimentally easiest discovery signature as the events should contain four isolated high $\mathrm{p}_{T}$ leptons. Furthermore, a Zmass constraint can be used for both lepton pairs to suppress other backgrounds. Estimates from ATLAS and CMS indicate that an integrated luminosity of about $10 \mathrm{fb}^{-1}$ is required to discover a SM Higgs in this mass range with at least 5 standard deviation [i $[\bar{L} \overline{8}]$. For example, an ATLAS study [1] ${ }_{1}$ ] shows that a Higgs $\left(\mathrm{m}_{H}=300 \mathrm{GeV}\right.$ and $H \rightarrow Z Z \rightarrow 4 \ell^{ \pm}$) should be seen with 35 signal events above a continuum background of $\approx 13 \pm 4$ events, assuming $10 \mathrm{fb}^{-1}$. This study indicates also that the signal-to-background rate can be significantly improved by requiring that one reconstructed $Z$ has a $\mathrm{p}_{T} \geq \mathrm{m}_{H} / 2$. Using this cut results in 13 signal events $\left(\mathrm{m}_{H}=300\right.$ $\mathrm{GeV})$ and a background of 0.6 events $\left(10 \mathrm{fb}^{-1}\right)$.

For Higgs masses above $\approx 400 \mathrm{GeV}$ additional signatures involving hadronic $W$ and $Z$ decays as well as invisible $Z$ decays like $H \rightarrow$ $Z Z \rightarrow \ell^{+} \ell^{-} \nu \bar{\nu}$ (see figure 10) have been investigated. The advantages of much larger branching ratios are however spoilt by larger backgrounds from $t \bar{t}, W+X$ and $Z+X$. These high mass Higgs signatures involve missing transverse energy and jet-jet masses and require thus hermetic detectors with good jet-energy reconstruction.

\subsubsection{The SM $H \rightarrow \gamma \gamma$ channel}

The $\gamma \gamma$ mass resolution depends upon the energy resolution and the resolution on the measured angle between the two photons. As regards the angle between the photons, the issue is the possible uncertainty on the knowledge of the position of the production vertex. Although very localised in the transverse plane, the interaction vertices have a r.m.s. spread of about $53 \mathrm{~mm}$ along the beam axis. If no other knowledge were available such a spread would contribute about $1.5 \mathrm{GeV}$ to the mass resolution. Detailed studies suggest that the correct vertex can be located using charged tracks, even at the highest luminosities, where there are on average nearly 20 inelastic interactions per bunch-crossing. This method of using tracks for the vertex localisation is based on the expectation that the Higgs production events are harder than minimum-bias events and that they contain more high $-\mathrm{p}_{T}$ tracks. Using this
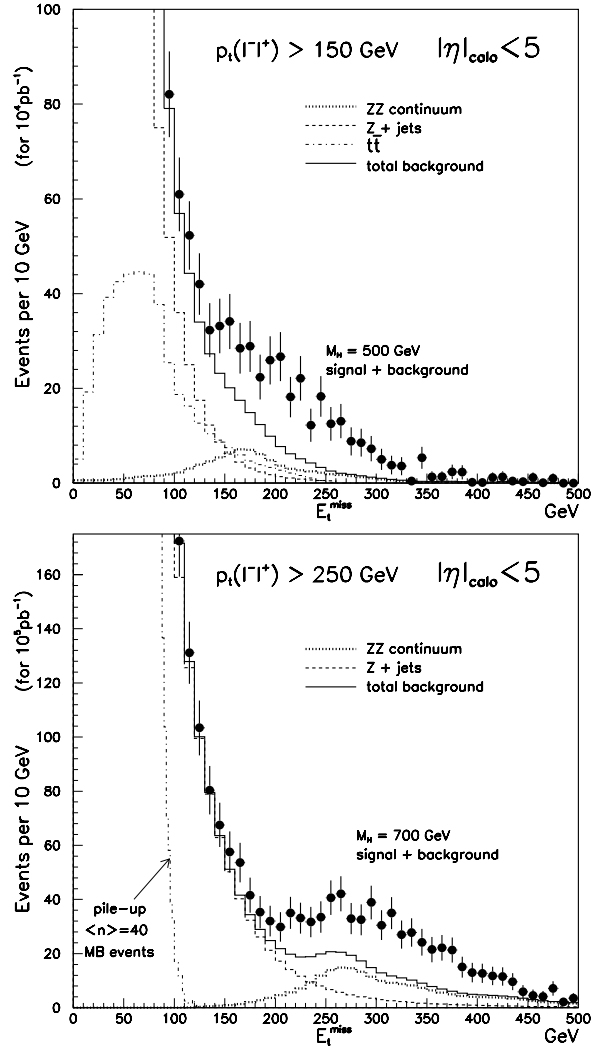

Figure 10: ATLAS simulation results for $H \rightarrow$ $Z Z \rightarrow \ell^{+} \ell^{-} \nu \bar{\nu}$.

fact it is possible to devise an algorithm to select the vertex of the Higgs event from the background of other primary vertices in the same bunch-crossing. This method is used by CMS [1 2 in]

ATLAS can use in addition the $1^{\text {st }}$ and $2^{\text {nd }}$ sampling of the electromagnetic calorimeter to measure the photon direction. In this case a contribution to the $\gamma \gamma$-mass resolution of about 530 $\mathrm{MeV}$ is expected [1]11].

Due to the material in front of the electromagnetic calorimeter (beam-pipe, inner tracking detector with support structures) photons will convert. In both experiments about $50 \%$ of the $H \rightarrow \gamma \gamma$ events have one or both of the photons converted. Detailed simulation studies have shown that a large fraction of these converted photons can be recovered with only a small degradation in resolution. Figure 11 shows the Higgs mass resolution taking these converted photons into account. This figure shows further that CMS expects about a factor 2 better mass resolution than ATLAS, which demonstrates the potential 


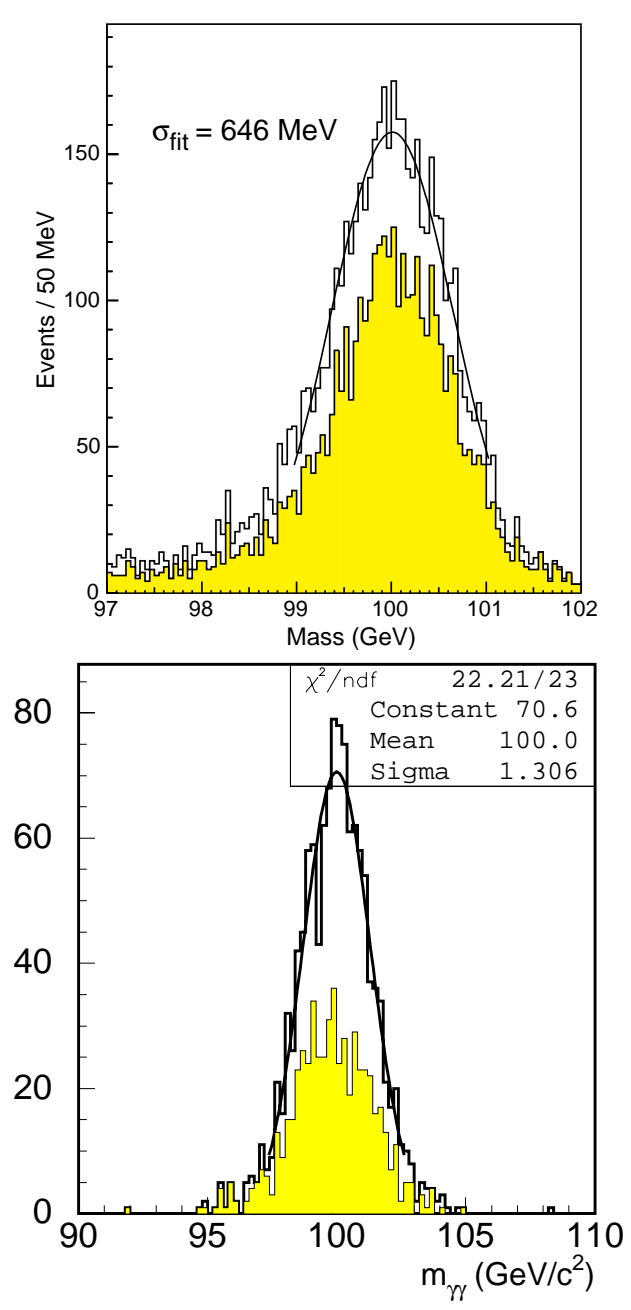

Figure 11: Higgs mass resolution including converted photons: in the top plot (CMS), the unconverted photons are shown as shaded area and in the bottom plot (ATLAS), the shaded area corresponds to the converted photons.

superior performance of a crystal calorimeter.

The dominant jet-background to the $H \rightarrow$ $\gamma \gamma$ signal comes from jet- $\gamma$ events, where the jet fragments to a leading $\pi^{0}$, carrying a large fraction of the jet transverse momentum. Isolation criteria using calorimeter and/or charged tracks are very powerful tools to reduce this potentially large background. In addition isolated $\pi^{0} \mathrm{~s}$ can be rejected by detecting the presence of two close-by electromagnetic showers rather than one. This can be achieved using the lateral shower shape of the electromagnetic cluster. The resulting rejection factor depends strongly on the $\pi^{0}$ transverse momentum, e.g. rejection factors larger than 3 for $\mathrm{p}_{T}<40 \mathrm{GeV}$ can be achieved with a small $\gamma$-efficiency loss. Figure 12 illustrates that isolation cuts together with a $\pi^{0}$-rejection algorithm reduce the $\gamma$-jet background well below the intrinsic $\gamma \gamma$ background.

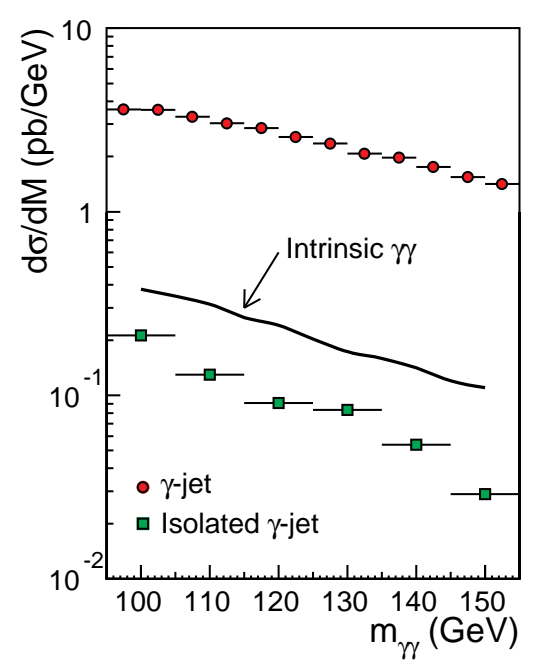

Figure 12: $\quad \gamma$-jet background cross-section as a function of mass before and after isolation. The line shows the level of the irreducible di-photon background expected in CMS.

The signal significance $\left(\mathrm{N}_{S} / \sqrt{N_{B}}\right)$ for a SM Higgs decaying to two photons has been evaluated using events within $\mathrm{a} \pm 1.4 \sigma$ mass window. Figure 13 shows the expected signal significance from CMS, as a function of the Higgs mass, for $30 \mathrm{fb}^{-1}$ and $100 \mathrm{fb}^{-1}$. This figure demonstrates further that a luminosity of $30 \mathrm{fb}^{-1}$ should enable CMS to detect the Higgs in the mass range between 100-150 GeV with more than five standard deviation in the decay $H \rightarrow \gamma \gamma$.

\subsubsection{The SM $H \rightarrow W^{+} W^{-} \rightarrow \ell^{+} \nu \ell^{-} \bar{\nu}$ channel}

A recent simulation has demonstrated that the $H \rightarrow W^{+} W^{-} \rightarrow \ell^{+} \nu \ell^{-} \bar{\nu}$ channel can be used to observe a statistically significant signal in the Higgs mass range of $130-200 \mathrm{GeV}$. This analysis [i $[1]$ ] exploits two important differences between a Higgs signal and the non-resonant background from $p p \rightarrow W^{+} W^{-} X$. The signal events from gluon-gluon scattering are more central than the $W^{+} W^{-}$background from $q \bar{q}$ scattering. This difference is exploited by the requirement that the polar angle $\theta$ of the reconstructed dilepton 


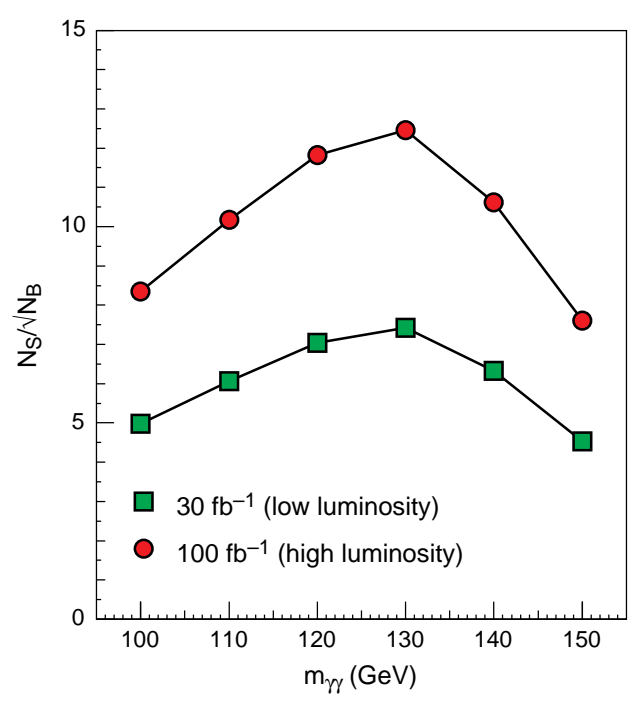

Figure 13: Signal significance as a function of $\mathrm{m}_{H}$, for $\mathrm{H} \rightarrow \gamma \gamma$ seen after $30 \mathrm{fb}^{-1}$ and $100 \mathrm{fb}^{-1}$ collected in CMS at low and high luminosity respectively.
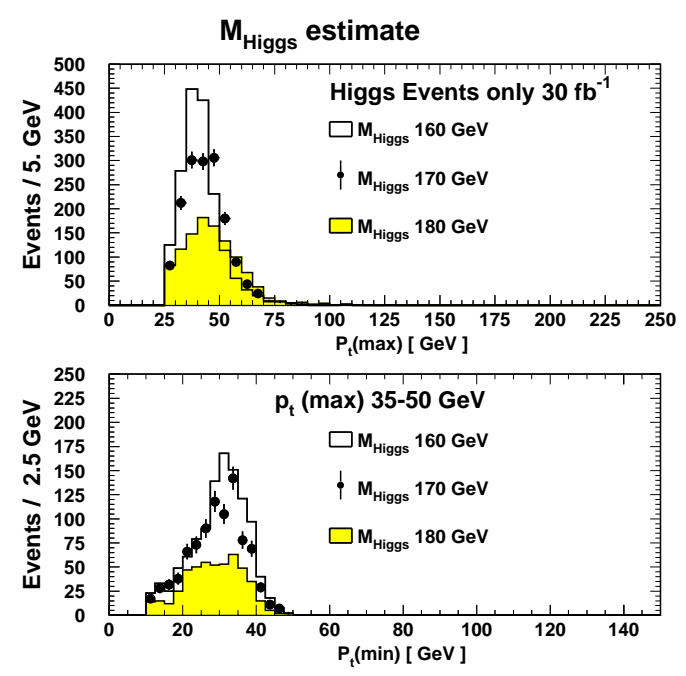

Figure 14: Expected lepton $\mathrm{p}_{T}$ spectra for $H \rightarrow$ $W^{+} W^{-} \rightarrow \ell^{+} \nu \ell^{-} \bar{\nu}$ and three different Higgs masses.

momentum vector, with respect to the beam direction, satisfies $|\cos \theta|<0.8$. As a result, both leptons are found essentially within the barrel region $(|\eta|<1.5)$ of the experiments. The $\cos \phi$ distribution shows the effect of $W^{+} W^{-}$spin correlations and the $\mathrm{V}-\mathrm{A}$ structure of the $W$ decays which results in a distinctive signature for $W^{+} W^{-}$pairs produced in Higgs decays. For a Higgs mass close to $2 \times \mathrm{m}_{W}$, the $\mathrm{W}^{ \pm}$boost is small and the opening angle between the two charged leptons in the plane transverse to the beam direction is very small.

The lepton $\mathrm{p}_{T}$ spectra, which are sensitive to the Higgs mass as shown in figure 14, can further be used to improve the signal to background ratio and to determine the Higgs mass with an accuracy of $\delta \mathrm{m}_{H} \approx \pm 5 \mathrm{GeV}$, assuming $5 \mathrm{fb}^{-1}$.

\subsection{SUSY Searches}

The attractive features of the MSSM are very well described in a Physics Report by H. P. Nilles [3] in 1984. We repeat here some of his arguments given in the introduction of the report:

"Since its discovery some ten years ago, Supersymmetry has fascinated many physicists. This has happened despite the absence of even the slightest phenomenological indication that it might be relevant for nature. .... Let us suppose that the Standard Model is valid up to a grand unification scale or even the Planck scale of $10^{19} \mathrm{GeV}$. The weak interaction scale of $100 \mathrm{GeV}$ is very tiny compared to these two scales. If these scales were input parameters of the theory the (mass) ${ }^{2}$ of the scalar particles in the Higgs sector have to be chosen with an accuracy of $10^{-34}$ compared to the Planck Mass. Theories where such adjustments of incredible accuracy have to be made are sometimes called unnatural.... Supersymmetry might render the Standard Model natural... To render the Standard Model supersymmetric a price has to be paid. For every boson (fermion) in the Standard Model, a supersymmetric partner fermion (boson) has to be introduced and to construct phenomenological acceptable models an additional Higgs supermultiplet is needed."

SUSY signatures are excellent benchmark processes to evaluate the physics performance of LHC detectors and they thus have influenced the detector optimisation. In order to cover the largest possible parameter space in the Higgs sector the searches are more challenging compared to the SM Higgs because: (i) one low mass Higgs (h) must exist, (ii) there are 5 Higgs bosons: $\mathrm{h}, \mathrm{H}^{0}$, $\mathrm{A}^{0}, \mathrm{H}^{ \pm}$and (iii) the expected $(\sigma \cdot \mathrm{BR})_{S U S Y}$ for the $\gamma \gamma$ - and $4 l$-channel are smaller than for the SM Higgs. For the simulation results discussed below, all sparticle masses are assumed to be 
heavy enough such that Higgs bosons decay only into SM particles.

In the sparticle sector many different signatures have been studied [20"] in the framework of the MSSM and mSUGRA. These studies include inclusive and exclusive signatures. Particular emphasis was given to the $\mathrm{E}_{T}^{\text {miss }}$ and b-jet signatures. In the following we briefly summarise the Higgs sector and discuss some selected topics in sparticle searches.

\subsubsection{The MSSM Higgs sector}

The MSSM Higgs sector requires the existence of two Higgs doublets, resulting in five physical Higgs bosons [2]1]. Within this model, at least one Higgs boson, $h$, should have a mass smaller than $\mathrm{m}_{h} \leq 125 \mathrm{GeV}[2 \overline{2} \overline{1}$. The upper mass limit depends via radiative correction on the top quark mass, the a priori unknown value of $\tan \beta$ and also via a mixing parameter on the mass of the stop quark. One expects that such a MSSM Higgs boson should be found soon at LEP200 if nature has chosen a $\tan \beta$ value smaller than 4 . The masses of the other four Higgs bosons $A, H^{0}$ and $H^{ \pm}$are less constrained but should essentially be degenerate once their mass is larger than about $200 \mathrm{GeV}$.

Current LHC studies show that the sensitivity to the MSSM Higgs sector is somehow restricted. This is illustrated in figure 15, where the sensitivity of different signatures is shown in a rather complicated two-dimensional multi-line contour plot.

The lightest neutral Higgs $h$. For the lightest Higgs the only established signature appears to be the decay $h \rightarrow \gamma \gamma$. For large masses of $\mathrm{m}_{A}\left(\mathrm{~m}_{A} \geq 400 \mathrm{GeV}\right)$ one finds essentially SM rates and sensitivity. For much smaller masses of $\mathrm{m}_{A}$, the branching ratio $h \rightarrow \gamma \gamma$ is too small to observe a statistically significant signal. The combination of the $h \rightarrow \gamma \gamma$ search with other $h$ decay modes, like $h \rightarrow b \bar{b}$, might help to enlarge the 5 sigma domain. A particular interesting aspect of an inclusive higgs search in SUSY events with the decay $h \rightarrow b \bar{b}$ decay will be discussed in the section on sparticle searches.

The heavy neutral Higgs Bosons $H^{0}, A^{0}$ and small values of $\tan \beta$. Assuming $\tan \beta$ values

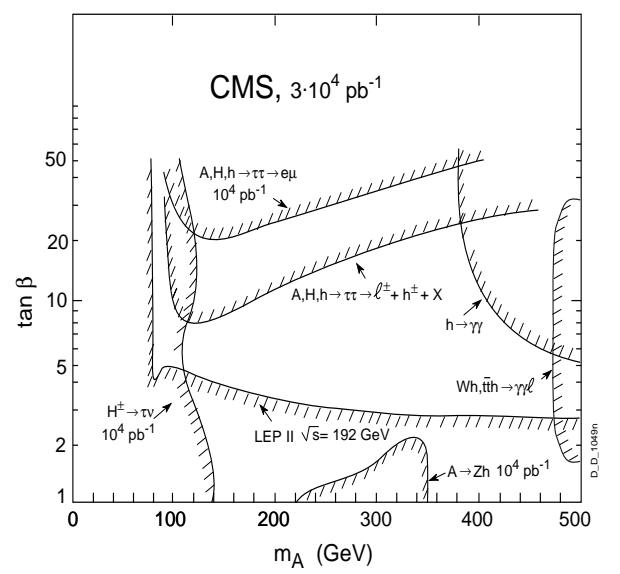

Figure 15: CMS 5 sigma significance contour plot for the MSSM Higgs sector in the $\mathrm{m}_{A}-\tan \beta$ plane. Each curve indicates the sensitivity for a specific Higgs search mode. No mixing in the stop sector is assumed.

below 4, for some $H^{0}$ masses and decays, significant signals might be visible. For example, a $H^{0}$ with a mass close to $170 \mathrm{GeV}$ appears to be detectable in the channel $H^{0} \rightarrow W W^{*} \rightarrow \ell \nu \ell \nu$. Other studies indicate the possibility to observe the decays $H^{0} \rightarrow h h \rightarrow \gamma \gamma b \bar{b}$ and $A \rightarrow Z h \rightarrow$ $\ell^{+} \ell^{-} b \bar{b}$ for masses between $200-350 \mathrm{GeV}$. We will not go into further details here, as the relevance of such studies for low values of $\tan \beta$ depend very strongly on the forthcoming LEP200 results.

The heavy neutral Higgs bosons $H^{0}, A^{0}$ and large values of $\tan \beta$. For large values of $\tan \beta$, the Higgs production cross sections, especially the ones for $b \bar{b} H^{0}$ and $b \bar{b} A^{0}$, are much larger than for the SM Higgs of similar mass. The only relevant Higgs decays are $H^{0}, A^{0} \rightarrow \tau \tau$ and $H^{0}, A^{0} \rightarrow b \bar{b}$.

Assuming large $\tan \beta$ values, the rare decay $A, H \rightarrow \mu \mu$ might show up as a resonance peak above a large background ${ }^{3}$. Assuming excellent mass resolution in the $\mu \mu$ channel of about $0.01-$ $0.02 \times \mathrm{m}_{\text {Higgs }}[\mathrm{in} \mathrm{GeV}$, a Higgs signal in this channel is detectable for an integrated luminosity of $30 \mathrm{fb}^{-1}$ and $\tan \beta \geq 20$.

\footnotetext{
${ }^{3}$ The branching ratio is expected to be about a factor of 300 smaller than the one for the decay to $\tau \tau$, as it scales with $\left(m_{\mu} / m_{\tau}\right)^{2}$.
} 
The charged Higgs $H^{ \pm}$. Depending only slightly on $\tan \beta$, the relevant charged Higgs decay modes are $H^{+} \rightarrow \tau^{+} \nu$ for masses below the $t \bar{b}$ threshold, and $H^{+} \rightarrow t \bar{b}$ above. Inclusive $t \bar{t}$ events might thus provide a good experimental signature for $H^{ \pm}$with a mass below $m_{t o p}-10$ $\mathrm{GeV}$. One has to search for $t \bar{t}$ events with isolated $\tau$ candidates which originate from the decay chain $t \bar{t} \rightarrow b W^{ \pm} b H^{ \pm}$and $H^{ \pm} \rightarrow \tau \nu$.

Another interesting process might be the production of a heavy $H^{ \pm}$in association with a top quark, $g b \rightarrow t H \rightarrow t t b \rightarrow W W b b b$. A parton level analysis of this channel [2$\overline{2}$ indicates the possibility to obtain $H^{ \pm}$mass peaks with reasonable signal-to-background ratios.

Summary for the MSSM Higgs sector. Figure 16 illustrates the sensitivity of the ATLAS experiment to various Higgs decay channels as discussed above [24]. CMS reports very similar results. These two-dimensional multi-line 5 sigma (statistical) significance plots, especially in the logarithmic version, indicate sensitivity over almost the entire MSSM parameter space. However, it is worth to remind the reader that

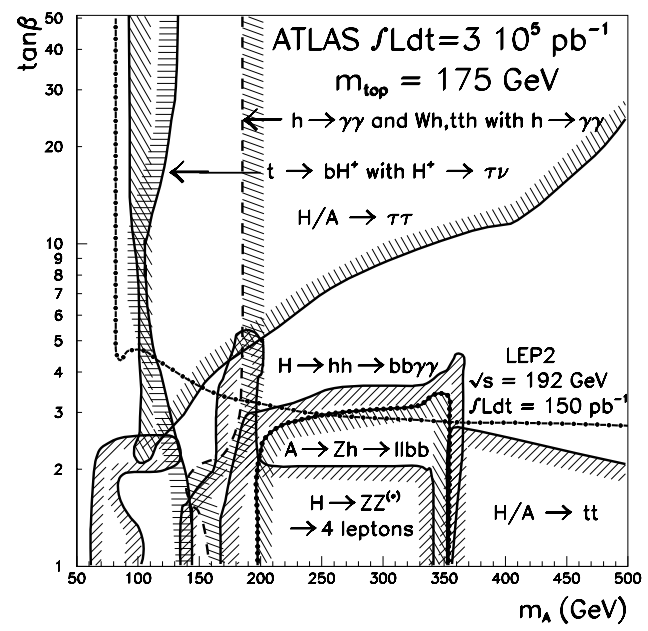

Figure 16: Estimated ATLAS sensitivity (5 sigma) for MSSM Higgs searches, assuming $300 \mathrm{fb}^{-1}$. The sensitivity of the different search signatures are shown in the $\mathrm{m}_{A}-\tan \beta$ plane.

only statistical errors and only decays into SM particles are included in the evaluation of the discovery potential. As a consequence, the obtained curves, especially when extrapolated to larger integrated luminosities and combined for ATLAS and CMS are doubtful. This is especially the case for channels like $W H \rightarrow \ell \nu b \bar{b}$ and for $H^{0}, A^{0} \rightarrow \tau \tau$ where the proposed signatures suffer certainly from the very poor signalto-background ratio.

\subsubsection{Searches for SUSY Particles}

The MSSM contains 124 free parameters including those of the SM. That many free parameters do not offer a good guidance for experimentalists. Additional assumptions to constrain the parameter space are therefore desirable. The simplest approach is the so called mSUGRA (minimal Super-Gravity) model with only five additional new parameters $\left(m_{0}, m_{1 / 2}, \tan \beta, A^{0}\right.$ and $\operatorname{sign}(\mu))$. This SUSY model is used for most of the simulation studies for which very advanced

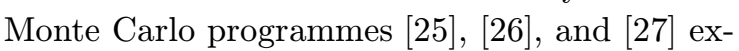
ist. This pragmatic choice of one approach to investigate the discovery potential appears to be sufficient, as essentially all required detector features can be tested.

\subsection{3 mSUGRA predictions}

Signatures related to the MSSM and in particular to mSUGRA searches are based on the consequences of R-parity conservation. R-parity is a multiplicative quantum number like ordinary parity. R-parity of the known SM particles is +1 , while it is -1 for sparticles. As a consequence, sparticles have to be produced in pairs. Sparticles decay either directly or via some cascade processes to SM particles and the lightest supersymmetric particle (LSP), which is a neutral, massive and stable object with neutrino-like properties. This LSP should have been abundantly produced after the Big Bang and is an excellent candidate for cold dark matter. Usually one assumes the LSP to be the lightest neutralino $\tilde{\chi}_{1}^{0}$ which escapes detection. Large missing transverse energy is thus the prime SUSY signature in collider experiments.

Within the mSUGRA model, the masses of sparticles are strongly related to the universal fermion and scalar masses $m_{1 / 2}$ and $m_{0}$. The masses of the spin- $-1 / 2$ sparticles are directly related to $m_{1 / 2}$. One expects approximately the following mass hierarchy: $\tilde{\chi}_{1}^{0} \approx 0.5 \cdot m_{1 / 2}, \tilde{\chi}_{2}^{0} \approx$ 
$\tilde{\chi}_{1}^{ \pm} \approx m_{1 / 2}$ and $\tilde{g} \approx 3 \cdot m_{1 / 2}$. The masses of the spin-0 sparticles are related to $m_{0}$ and $m_{1 / 2}$ and allow for some mass splitting between the "left" and "right" handed scalar partners of the degenerated left and right handed fermions. One finds the following simplified mass relations:

$m(\tilde{q})(\tilde{u}, \tilde{d}, \tilde{s}, \tilde{c}$ and $\tilde{b}) \approx \sqrt{m_{0}^{2}+6 \cdot m_{1 / 2}^{2}}$

$m(\tilde{\nu})_{l e f t} \approx m\left(\tilde{\ell^{ \pm}}\right)_{l e f t} \approx \sqrt{m_{0}^{2}+0.52 \cdot m_{1 / 2}^{2}}$

and $m\left(\tilde{\ell^{ \pm}}\right)_{\text {right }} \approx \sqrt{m_{0}^{2}+0.15 \cdot m_{1 / 2}^{2}}$.

The masses of the left and right handed stop quarks $\left(\tilde{t}_{\ell, r}\right)$ can have a large splitting. As a result, the right handed stop quark might be the lightest of all squarks.

Following the above mass relations and using the known SUSY couplings, possible SUSY decays and the related signatures can be calculated.

As an example we consider the $\tilde{\chi}_{2}^{0}$ decay $\tilde{\chi}_{2}^{0} \rightarrow$ $\tilde{\chi}_{1}^{0}+X$ with $X$ being: $\gamma^{*} Z^{*} \rightarrow \ell^{+} \ell^{-}$or $h^{0} \rightarrow b \bar{b}$ or $Z \rightarrow f \bar{f}$. Other possible $\tilde{\chi}_{2}^{0}$ decay chains are $\tilde{\chi}_{2}^{0} \rightarrow \tilde{\chi}_{1}^{ \pm(*)}+\ell^{\mp} \nu$ and $\tilde{\chi}_{1}^{ \pm(*)} \rightarrow \tilde{\chi}_{1}^{0} \ell^{ \pm} \nu$ or $\tilde{\chi}_{2}^{0} \rightarrow \tilde{\ell}^{ \pm} \ell^{\mp}$. This example clearly demonstrates the complexity of sparticle signatures.

For higher sparticle masses, even more decay channels might open up. It is thus not possible to define all search strategies a priori. Furthermore, possible unconstrained mixing angles between neutralinos lead to a model dependent search strategy for squarks and gluinos, as will be discussed below.

The discovery potential is usually given in the $m_{0}-m_{1 / 2}$ parameter space. Despite the model dependence, it allows to compare the sensitivity of different signatures. Having various proposed methods, the resulting figures are - as in the Higgs sector - rather complicated and require some time for appreciation. A typical example is shown in figure 17 [2] curves indicate the LHC sensitivity for different signatures and different sparticles. It is usually assumed that the maximum information about SUSY can be extracted in regions which are covered by many different signatures. The meaning of the various curves should become clear in the following section.

\subsubsection{Squark and Gluino searches}

The cross-section for strongly interacting spar-
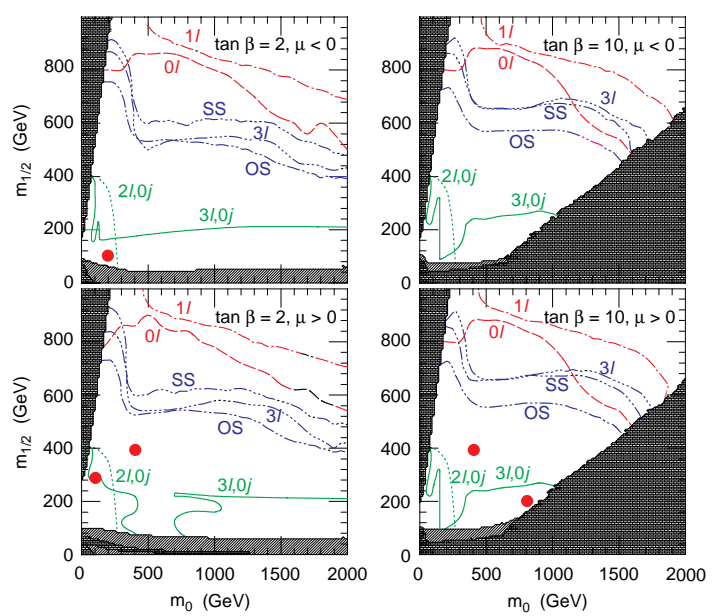

Figure 17: Expected mSUGRA sensitivity of various signatures in the $m_{0}-m_{1 / 2}$ plane at the LHC, assuming an integrated luminosity of $10 \mathrm{fb}^{-1}$. The different curves indicate the expected sensitivity for SUSY events with $\mathrm{n}$ leptons $(\ell)$ and for events with lepton pairs with same charge (SS) and opposite charge (OS).

ticles are large at the LHC, as can be seen in figure 18 : $2 \overline{8}$. For example the pair production cross-section of squarks and gluinos with a mass of $\approx 1 \mathrm{TeV}$ has been estimated to be as large as $1 \mathrm{pb}$ resulting in $10^{4}$ produced SUSY events for $10 \mathrm{fb}^{-1}$. This high rate, combined with the possibility to observe many different decay modes, could turn LHC into a "SUSY-factory".

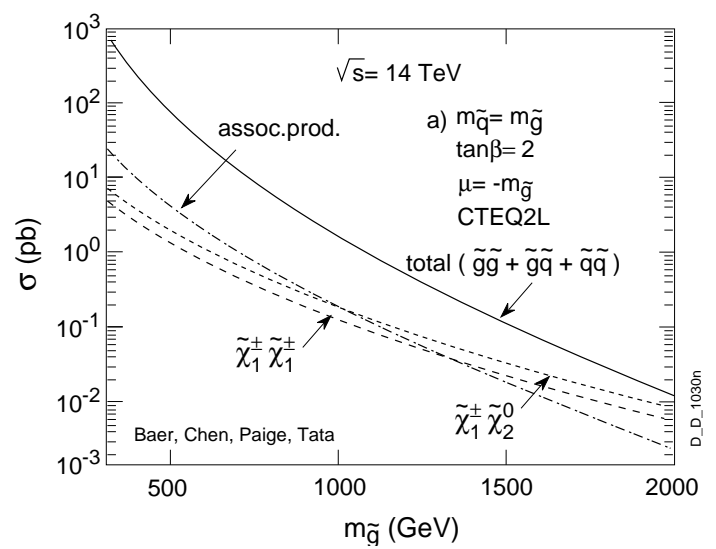

Figure 18: Cross-sections for sparticle production at the LHC [28i].

Depending on the SUSY model parameters, a large variety of massive squark and gluino decay channels and signatures might exist. A search for squarks and gluons at the LHC should con- 
sider the various signatures resulting from the following decay channels:

- $\tilde{g} \rightarrow \tilde{q} \bar{q}$ including $\tilde{g} \rightarrow \tilde{t} \bar{t}$

- $\tilde{q} \rightarrow \tilde{\chi}_{1}^{0} q \quad$ or $\quad \tilde{q} \rightarrow \tilde{\chi}_{2}^{0} q \quad$ or $\quad \tilde{q} \rightarrow \tilde{\chi}_{1}^{ \pm} q^{\prime}$

- $\tilde{\chi}_{2}^{0} \rightarrow \ell^{+} \ell^{-} \tilde{\chi}_{1}^{0} \quad$ or $\quad \tilde{\chi}_{2}^{0} \rightarrow Z^{0} \tilde{\chi}_{1}^{0} \quad$ or $\tilde{\chi}_{2}^{0} \rightarrow h^{0} \tilde{\chi}_{1}^{0}$

- $\tilde{\chi}_{1}^{ \pm} \rightarrow \tilde{\chi}_{1}^{0} \ell^{ \pm} \nu \quad$ or $\quad \tilde{\chi}_{1}^{ \pm} \rightarrow W \tilde{\chi}_{1}^{0}$.

The various decay channels can be separated into at least three distinct event signatures.

- Multi-jets + missing transverse energy; these events should be spherical in the plane transverse to the beam.

- Multi-jets + missing transverse energy + $\mathrm{n}(=1,2,3,4)$ isolated high $\mathrm{p}_{T}$ leptons; these leptons originate from cascade decays of charginos and neutralinos.

- Multi-jets + missing transverse energy + lepton pairs of the same charge; such events can originate from $\tilde{g} \tilde{g} \rightarrow \tilde{u} \bar{u} \tilde{d} \bar{d}$ with subsequent decays of the squarks to $\tilde{u} \rightarrow \tilde{\chi}^{+} d$ and $\tilde{d} \rightarrow \tilde{\chi}^{+} u$ followed by leptonic chargino decays $\tilde{\chi}^{+} \rightarrow \tilde{\chi}_{1}^{0} \ell^{+} \nu$.

The observation and detailed analysis of different types of SUSY events might allow the discovery of many sparticles and should help to measure some of the SUSY parameters.

A search strategy for squarks and gluinos at the LHC would select jet events with large visible transverse mass and missing transverse energy. Such events can then be classified according to the number of isolated high $\mathrm{p}_{T}$ leptons including the lepton flavour and charge relation. Once an excess above SM backgrounds is observed, one would try to interpret the events and measured cross-section(s) in terms of $\tilde{g}, \tilde{q}$ masses and decay modes for various SUSY models. Concerning the SM background processes, the largest backgrounds originate mainly from $W+$ jet(s), $Z+$ jet(s) and $t \bar{t}$ events. Using this approach, very encouraging signal-to-background ratios, combined with sizable signal cross-sections, are obtainable for a large range of squark and gluino masses. The simulation results of such studies indicate, as shown in figure 19, that LHC

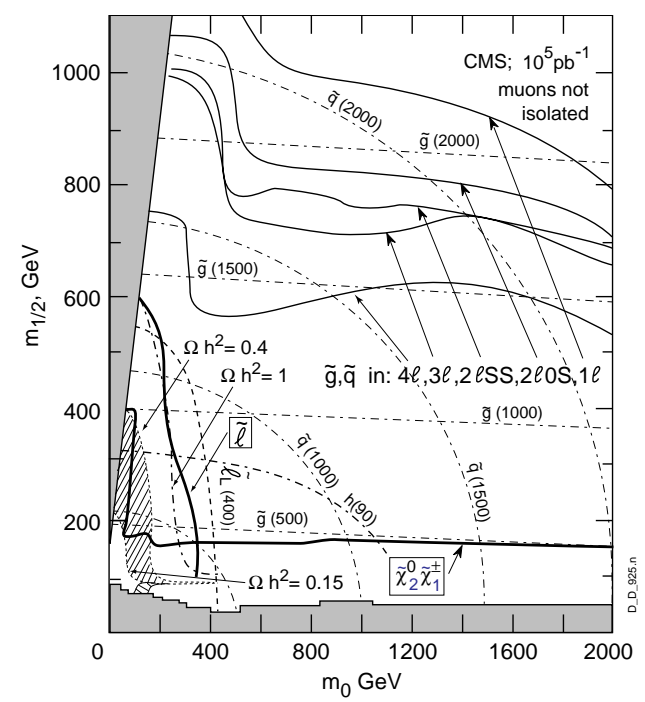

Figure 19: Expected CMS sensitivity for squarks and gluinos, sleptons and for $\tilde{\chi}_{2}^{0} \tilde{\chi}_{1}^{ \pm}$in the $m_{0}-$ $m_{1 / 2}$ plane, assuming $100 \mathrm{fb}^{-1}\left[{ }^{-1} 9^{\prime}\right]$. The different solid lines show the expected 5 sigma signal, estimated from $\mathrm{S} / \sqrt{S+B_{S M}}$, coverage domain for the various signatures using isolated high $\mathrm{p}_{T}$ leptons. The dashed-dotted lines indicate the corresponding squark and gluino masses.

experiments are sensitive to squark and gluinos masses up to about $2 \mathrm{TeV}$, assuming $100 \mathrm{fb}^{-1}$ [2 $\left.2 \overline{9}_{1}^{7}\right]$. Figure 19 also illustrates that detailed studies of branching ratios using the different decay chains are possible up to squark or gluino masses of about $1.5 \mathrm{TeV}$, where significant signals can be observed for many different channels. Another consequence of the expected large signal crosssections is the possibility that at the LHC startup, for an integrated luminosity of only $\approx 100$ $\mathrm{pb}^{-1}$, squarks and gluinos up to masses of about 600 to $700 \mathrm{GeV}$ can be discovered, which is well beyond the most optimistic Tevatron Run III accessible mass range.

Given this exciting squark and gluino discovery potential for many different channels, one has to keep in mind that all potential signals depend strongly on the understanding of the various background processes and thus on the detector systematics. A thorough study of backgrounds including the shapes of background distributions is therefore mandatory. In particular, the requirements of very efficient lepton identifi- 
cation and good missing transverse energy measurement demand for a well understood detector response. This will require certainly some time, given the complexity of the LHC detectors.

Our discussion of the SUSY discovery potential has illustrated the sensitivity of the proposed ATLAS and CMS experiments. The next step after a discovery is the determination of SUSY parameters, thus obtaining a deeper insight of the underlying theory.

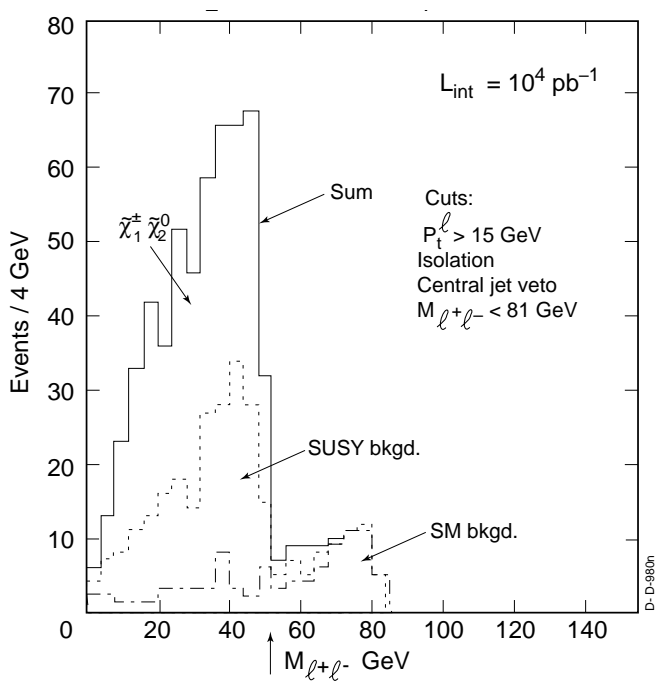

Figure 20: Expected dilepton mass distribution in CMS for $\mathrm{L}=10 \mathrm{fb}^{-1}$ using trilepton events from $\tilde{\chi}_{2}^{0} \tilde{\chi}_{1}^{ \pm}$ decays [29]. The upper edge in the distribution at about $50 \mathrm{GeV}$ corresponds to the kinematic limit in the decay $\tilde{\chi}_{2}^{0} \rightarrow \ell^{+} \ell^{-} \tilde{\chi}_{1}^{0}$ and is thus sensitive to the mass difference of $\tilde{\chi}_{2}^{0}-\tilde{\chi}_{1}^{0}$.

The production and decays of $\tilde{\chi}_{2}^{0} \tilde{\chi}_{1}^{ \pm}$provide enough rates for masses $\leq 200 \mathrm{GeV}$ and should allow, as shown in figure 20, to measure the dilepton mass distribution and their relative $\mathrm{p}_{T}$ spectra. The mass distribution and especially the edge in the mass distribution has been shown to be sensitive to the mass difference between the two neutralinos involved in the decay.

In contrast to the rate limitations of weakly produced sparticles at the LHC, detailed studies of clean squark and gluino events are expected to reveal more information. One finds that the large rate for many distinct event signatures allows to measure masses and mass ratios for several SUSY particles, produced in cascade decays of squarks and gluons. Many of these ideas have been discussed at the 1996 CERN SUSY-Workshop [30'].
An especially interesting proposal is the detection of the Higgs boson, $h$, via the decay chain $\tilde{\chi}_{2}^{0} \rightarrow \tilde{\chi}_{1}^{0} h \rightarrow \tilde{\chi}_{1}^{0} b \bar{b}$. Higgs mass peaks above background are found for various choices of $\tan \beta$ and $m_{0}, m_{1 / 2}$.

Another interesting approach to determine the SUSY mass scale has been suggested in $3 \overline{1}$. The idea is to define an effective mass, using the scalar $\mathrm{p}_{T}$ sum of the four jets with the largest transverse energy plus the missing transverse energy of the event. This effective mass shows an approximately linear relation with the underlying SUSY mass-scale, defined as the minimum of the squark or gluino mass.

The possibility to extract model parameters within the mSUGRA framework was illustrated using the following method: for each point in the parameter space a set of experimental constraints on sparticle masses is derived and a fit to parameters of mSUGRA is performed. For example, assuming $\mathrm{m}_{0}=\mathrm{m}_{1 / 2}=400 \mathrm{GeV}, \mathrm{A}_{0}=0, \tan \beta$ $=2$ and $\operatorname{sign}(\mu)=+$, one obtains for $30 \mathrm{fb}^{-1}$ the following sensitivity: $\mathrm{m}_{0}=400 \pm 100 \mathrm{GeV}, \mathrm{m}_{1 / 2}$ $=400 \pm 10 \mathrm{GeV}$ and $\tan \beta=2 \pm 0.8$.

\section{Concluding Remarks}

The LHC is currently the only realistic possibility to reach the $\mathrm{TeV}$ energy range in constituentconstituent scattering; this energy range is expected to be rich in discoveries.

A large international community is working on the realization of the LHC experimental programme. The two large general-purpose detectors ATLAS and CMS have moved from the R\&D to preproduction and in certain areas to the production phase. The concept of both detectors was driven by physics considerations using SM and beyond the SM processes, which were used to optimise the final detector design.

It was already pointed out that, although the most exciting discoveries will be those of totally unexpected new particles or phenomena, one can only demonstrate the discovery potential of the proposed experiments using predicted new particles. However, the experiments designed under these considerations should also allow the discovery of whatever new phenomena might occur in multi-TeV pp collisions. 


\section{Acknowledgments}

I would like to thank the organizers for the invitation to this very interesting school. I also want to thank Michael Dittmar for his valuable contributions to this paper.

\section{References}

[1] For a review of the experimental status of the SM see D. Treille, these proceedings.

[2] For a review and further references see: The Higgs Hunter Guide, UCD-89-4, SCIPP-89/13, BNL-41644.

[3] H.P. Nilles, Phys. Reports 110, 1 (1984).

[4] A. Dobado et al. , Proc. LHC Workshop, Aachen, 1990, CERN 90-10(1990), Vol.II; R.Casalbuoni et al. , ibid.

E. Eichten et al., Phys. Lett. B 405, 305 (1997).

[5] The LHC Study Group, The Large Hadron Collider: Conceptual Design, CERN/AC/9505(LHC), 1995.

[6] T. Hambye and K. Riesselmann, Phys. Rev. D 55, 7255 (1997) and hep-ph/9708416.

[7] Open presentation of the four LEP Collaborations at the LEPC meeting, CERN, 12 November 1998 .

[8] See for example Günter Quast, LEPEWWG Status Report at the LEPC meeting, 15 September 1998 .

[9] See for example E. Gross, A.L. Read and D. Lellouch, CERN-EP/98-094 (1998).

[10] Recent search results are summarised in: D. Treille, Plenary Talk at ICHEP 98, Vancouver, British Columbia, Canada, July 23-30, 1998, to appear in the proceedings

[11] ATLAS Collaboration, Technical Proposal, CERN/LHCC 94-43, LHCC/P2, 15 December 1994; The Muon Spectrometer Technical Design Report, CERN/LHCC 97-22, 5 June 1997; Tile Calorimeter Technical Design Report, CERN/LHCC 96-42, 15 December 1996; Calorimeter Performance Technical Design Report, CERN/LHCC 96-40, 13 January 1997; Liquid Argon Calorimeter Technical Design Report, CERN/LHCC 96-41, 15 December 1996; Inner Detector Technical Design Report, CERN/LHCC 97-16 and 97-17, 30 April 1997.
[12] CMS Collaboration, Electromagnetic Calorimeter Project, Technical Design Report, CERN/LHCC 97-33, 15 December 1997; Technical Proposal, CERN/LHCC 94-38, LHCC/P1, 15 December 1994; The Muon Project Technical Design Report, CERN/LHCC 97-32, 15 December 1997; The Hadron Calorimeter Project Technical Design Report, CERN/LHCC 97-31, 20 June 1997; The Tracker Project Technical Design Report, CERN/LHCC 98-6, 15 April 1998.

[13] F. Pauss and M. Dittmar, Experimental Challenges at the LHC, to appear in the Proceedings of the ASI Summer School, St.Croix, 1998 and ETHZ-IPP PR-98-09

[14] Z. Kunszt, S. Moretti and W. J. Stirling; Z. Phys. C 74, 479 (1997) and hep-ph/9611397.

[15] The large Higgs production rate from gluongluon fusion has originally been pointed out by H. Georgi, S. Glashow, M. Machacek and D. Nanopoulos; Phys. Rev. Lett. 40, 692 (1978).

[16] The assumed Higgs cross section (NLO) are taken from i14.1] while the branching ratios are taken from the program HDECAY written by A. Djouadi, J. Kalinowski and M. Spira; e-Print Archive: hep-ph/9704448.

[17] M. Dittmar and H. Dreiner; Phys. Rev. D 55, 167 (1997) and hep-ph/9608317.

[18] Justifications about the expected performance figures can be found at http://atlasinfo.cern.ch/Atlas/Welcome.html for ATLAS and at http://cmsdoc.cern.ch/cms.html for CMS.

[19] E. Richter-Was et al. ATLAS Internal Note Phys-No-048, 17/7/1995 (unpublished).

[20] For a summary of early studies of SUSY signatures at the LHC see: F. Pauss, Proc. LHC Workshop, Aachen, 1990, CERN 90-10(1990), Vol.I.

[21] For a summary on supersymmetric Higgs searches at the LHC and a discussion of the notation see: Z. Kunszt and F. Zwirner, Proc. LHC Workshop, Aachen, 1990, CERN 9010(1990), Vol.II.

[22] For a recent calculation of the upper limit for the lightest MSSM Higgs mass and further references therein see: M. Quiros and J. R. Espinosa CERN-TH-98-292, Sep 1998 and hep$\mathrm{ph} / 9809269$. 
[23] V. Barger, R.J.N. Phillips and D.P. Roy; Phys. Lett. B 324, 236 (1994) and hep-ph/9311372.

[24] E. Richter-Was et al. ; Int. J. Mod.Phys. A13, 1371 (1998) and CERN-TH-96-111.

[25] ISAJET 7.40, F. E. Paige, S. D. Protopescu, H. Baer and X. Tata; BNL-HET-98-39, and hep$\mathrm{ph} / 9810440$

[26] SPYTHIA, S. Mrenna; Comput. Phys. Commun. 101, 232 (1997) and e-Print Archive: hep$\mathrm{ph} / 9609360$

[27] T. Sjöstrand, Comput. Phys. Commun. 82, 74 (1994).

[28] H. Baer, C. Chen, F. Paige and X. Tata; Phys. Rev. D 53, 6241 (1996) and hep-ph/9512383.

[29] S. Abdullin et al. ; CMS Note-1998/006.

[30] For an overview of LHC SUSY studies see the transparencies of the 1996 LHCC SUSY Workshop, October 29-30; CMS document 1996-149 Meeting.

[31] I. Hinchliffe, F. Paige, G. Polesello and E. Richter-Was; ATLAS-Phys-No-107. 